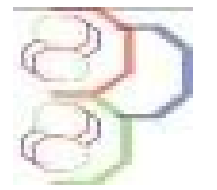

Journal of Applied Biosciences 146: 15074 - 15080

ISSN 1997-5902

\title{
Rendement carcasse des ovins sahéliens dans la Province du Guera au Centre-Est du Tchad.
}

\author{
Mopaté Logténé Youssouf1, Madjina Tellah²@, Issa Youssouf Adoum 2, Mahamat Seid souleyman², \\ 1 Institut de Recherche en Elevage pour le Développement (IRED), BP : 433 N'Djaména \\ 2 Institut National Supérieur des Sciences et Techniques d'Abéché (INSTA), BP : 130 Abéché \\ Adresse de l'auteur correspondant : madjinatellah@gmail.com
}

Original submitted in on $22^{\text {nd }}$ October 2019. Published online at www.m.elewa.org/journals/ on 29th February 2020

https://doi.org/10.35759/JABs.v146.10

\section{RESUME}

Objectif: évaluer le rendement carcasse des ovins sahéliens afin d'orienter les choix des actions de développement de la production de viande ovine dans la Province du Guera au Centre-Est du Tchad.

Méthodologie et résultats : les poids des ovins avant abattage, poids des carcasses à chaud et à froid ont été mesurés. Les pesées ont été effectuées trois fois par semaine d'octobre à décembre 2018. L'étude a concerné 192 ovins dont 127 ovins abattus à l'abattoir municipal de Mangalmé et 65 têtes abattus à celui de Mongo. Ces ovins provenaient surtout pour $86 \%$ des élevages transhumants contre $14 \%$ des élevages sédentaires. Soixante-dix-sept mâles soit $40 \%$ ont été âgés de $28,9 \pm 22,6$ mois avec un poids moyen à l'abattage de 24,1 $\pm 8,2 \mathrm{~kg}$ et 115 femelles soit $60 \%$, âgées de $42,4 \pm 31,6$ mois avec un poids de $32,5 \pm 8,6 \mathrm{~kg}$. La majorité des ovins abattus (environ $85 \%$ ) à Mangalmé et à Mongo provient des élevages transhumants. Les brebis ont été plus lourdes et plus âgées à l'abattage que les béliers. Le rendement carcasse des brebis a été inférieur à celui des carcasses des boucs. L'abattage des brebis occasionne de pertes en reproduction des brebis dans la zone d'étude. L'origine des ovins abattus n'a pas eu d'influence sur le rendement carcasse des brebis. Chez les mâles provenant d'élevage sédentaire, le rendement carcasse a été meilleur que celui béliers sédentaires. Conclusion et application des résultats : l'abattage des béliers est à encourager pour limiter des pertes en reproduction des femelles et améliorer l'approvisionnement en viande de la population. Ce résultat suggère d'orienter et d'encourager les éleveurs dans la production des agneaux de boucherie. Pour cela une transition du système extensif transhumant à un mode d'élevage intensif et spécialisé est conseillée. Les brebis épargnées de l'abattage induiront une amélioration de la productivité des troupeaux. Ce mode d'élevage permettra également de mieux valoriser les ressources alimentaires locales, d'améliorer la qualité de la viande produite et de maîtriser la reproduction ovine en zone sahélienne du Tchad.

Mots clés : Ovin sahélien, Rendement carcasse, Centre-Est, Tchad.

\begin{abstract}
Objective: to assess the carcass yield of Sahelian sheep in order to guide the choices for actions to develop sheep meat production at the Province of Guera in Central-Eastern Chad.

Methodology and results: sheep weights before slaughter, carcass weights at hot and cold were measured. The weighing was carried out three times a week from October to December 2018. The study concerned 192 sheep including 127 sheep slaughtered at the municipal slaughterhouse of Mangalme and 65 heads slaughtered at that of Mongo. These sheep originated mainly for $86 \%$ of transhumant breeding against $14 \%$ of
\end{abstract}


sedentary livestock. Seventy-seven males (40\%) were aged $28.9 \pm 22.6$ months with an average slaughter weight of $24.1 \pm 8.2 \mathrm{~kg}$ and 115 females $(60 \%)$ aged $42.4 \pm 31.6$ months with a weight of $32.5 \pm 8.6 \mathrm{~kg}$. The majority of sheep slaughtered (around $85 \%$ ) in Mangalmé and Mongo come from transhumant herds. Ewes were heavier and older at slaughter than rams. The carcass yield of the ewes was lower than that of the goat carcasses. The slaughter of the ewes causes losses in ewe's reproduction at the study area. The origin of the slaughtered sheep did not affect the carcass yield of the ewes. In males from sedentary breeding, the carcass yield was better than that of sedentary rams.

Conclusion and application of results: the slaughter of rams is to be encouraged to limit losses in female reproduction and improve the population's meat supply. This result suggests to guide and encourage breeders in the production of slaughter lambs. For this, a transition from the extensive transhumant system to an intensive and specialized farming method is recommended. Sheep spared from slaughter will improve herd productivity. This farming method will also make it possible to better develop local food resources, improve the quality of the meat produced and control sheep reproduction in the Sahelian zone of Chad.

Key words: Sahelian Sheep, Carcass Yield, Centre-East, Chad.

\section{INTRODUCTION}

Le cheptel tchadien est composé surtout de bovins, ovins, caprins et camélidés pour un effectif de 93803192 têtes suivant le recensement général du bétail (MERA, 2015). Ces ruminants dictent la vocation pastorale du Tchad et sont d'une importance capitale pour l'économie du pays. Les petits ruminants et plus particulièrement les ovins sont les espèces animales les plus élevées en zones tropicale et sahélienne. Ils jouent un rôle très important dans la sécurité alimentaire; et contribuent fortement à l'économie familiale et la réduction de la pauvreté en milieu rural (Alary et al., 2015). Ils sont particulièrement sollicités lors des cérémonies et rituels musulmans. Les modes d'élevage (nomade, transhumant et sédentaire) des ovins épousent les trois grandes zones écologiques (saharienne, sahélienne et soudanienne) du Tchad. Les ressources alimentaires (fourrages et eau) dictent l'adoption de chacun de ces modes de conduite. Elles expliquent aussi les variables de sortie de ces systèmes notamment les performances pondérales (poids vif et rendements carcasse) des animaux qui évoluent dans ces zones. A l'exception des caprins (Mopaté et al., 2019), peu d'études spécifiques sur les rendements carcasse des petits ruminants sont disponibles au Tchad. Chez les caprins sahéliens commercialisés dans la Province du Guéra, les rendements carcasse ont varié selon les sexes des sujets abattus (Mopaté et al., 2019). Chez les ovins sahéliens en revanche, les rendements carcasse variables selon les races ont été observés (Dumas, 1980). Toutefois, les ovins sahéliens sont connus pour leur production de viande avec des caractéristiques suivantes: un poids vif de 43,94 $\pm 7,18 \mathrm{~kg}$, un poids carcasse chaude de $23,19 \pm 4,77 \mathrm{~kg}$ et un poids carcasse froide de $21,25 \pm 4,37 \mathrm{~kg}$ et un rendement carcasse qui varie de $52,59 \pm 3,89$ à $60,28 \pm 4,48 \%$ suivant l'état de refroidissement de la carcasse (Djenontin et al., 2017). II est à signaler que l'abattage des femelles gravides suite à un accroissement de la demande en protéine ou par ignorance des producteurs, entraine des pertes en reproduction (petits et femelles en âge de reproduction) non négligeables mais très peu prises en compte (Idahor, 2013; Alade et al., 2011; Garba et al., 1992). Or au Cameroun, la perte due à l'abattage des chèvres gravides a été de l'ordre $7 \%$ de l'effectif des femelles (Nana et al., 2014). L'objet de l'étude a été d'estimer les rendements carcasse des ovins sahéliens abattus aux abattoirs des villes de Mongo et de Mangalmé, d'orienter les choix des actions de développement et d'évaluer indirectement l'importance de la transhumance dans l'approvisionnement en viande de ces villes dans le Nord-Guéra, au Centre-Est du Tchad. 

du Tchad.

\section{MATERIEL ET METHODES}

Zone d'étude : L'étude a été réalisée dans deux villes dont Mongo et Mangalmé situées dans la Province du Guera. Les localisations par coordonnées GPS (Global Positioning System) sont : $12^{\circ} 09^{\prime} 30^{\prime \prime}$ de latitude Nord et $18^{\circ} 40^{\prime} 30^{\prime \prime}$ de longitude Est pour la ville de Mongo et $12^{\circ}$ $21^{\prime} 04^{\prime \prime}$ et $19^{\circ} 36^{\prime} 01^{\prime \prime}$ pour celle de Mangalmé.
Echantillonnage et méthodes de collecte de données : L'étude a concerné 192 ovins acquis par les bouchers sur le marché dont 127 ovins à Mangalmé et 65 ovins à Mongo (Tableau 1).

Tableau 1: Effectifs des ovins abattus dans les abattoirs municipaux de Mangalmé et Mongo au Centre du Tchad selon le sexe et l'origine des animaux (sédentaire ou transhumant).

\begin{tabular}{llll}
\hline Sexe & Elevage sédentaire & Elevage transhumant & Effectif (n) \\
\hline Mâle & 15 & 62 & 77 \\
Femelle & 12 & 103 & 115 \\
\hline Total $(\mathrm{N})$ & 27 & 165 & 192 \\
\hline
\end{tabular}

Les informations collectées avant l'abattage des ovins concernaient, le sexe, les origines des animaux (sédentaires ou transhumances), l'âge en mois estimé selon la dentition, l'état corporelle, le poids vif après un jeûne de 24 heures. Après l'abattage, la saignée, l'éviscération et le dépeçage, les données collectées ont portées sur l'état de gestation des femelles observées par examen de l'utérus et la pesée des carcasses à chaud (immédiatement après la saignée, le dépouillement, l'éviscération et l'enlèvement de la tête, des pattes, des mamelles, des organes génitaux). Le poids carcasse à chaud a été retenu afin de libérer rapidement les bouchers pour le marché. Les pesés ont été réalisés à l'aide des pesons de $50 \mathrm{~kg}$, avec un mini de $1 \mathrm{~kg}$ et $\mathrm{d}=500 \mathrm{~g}$. Les données ont été collectées trois fois par semaine et pendant trois mois (d'octobre à décembre 2018).

\section{RESULTATS}

Effectifs, sexe et origine des ovins abattus: Sur l'ensemble des ovins abattus, $66 \%$ l'ont été l'abattoir de

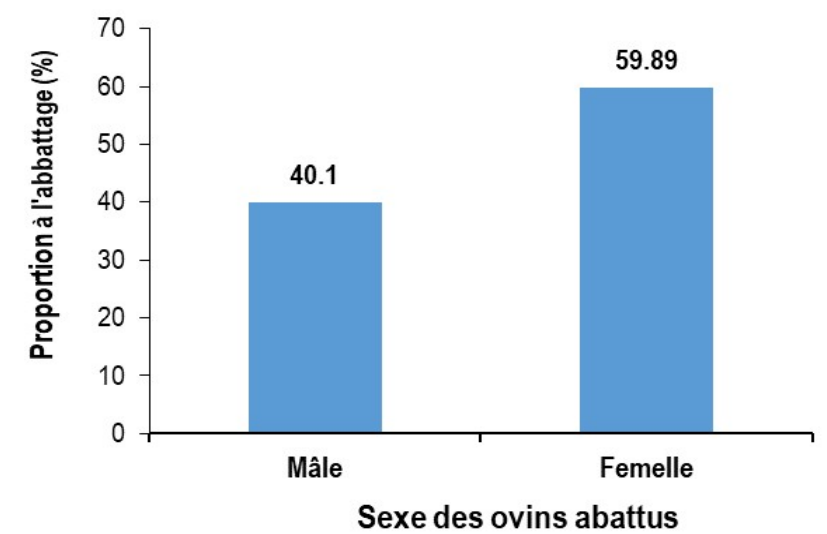

Analyse des données : Les informations recueillies ont été saisies avec le logiciel de base de données "Accès», puis transférées sous le logiciel de traitement «Winstat i.c» (Cirad/IITCF). L'analyse de variance a été faite et la signification sur les différences des moyennes a été recherchée au seuil de $5 \%$. Le poids à froid a été déduit de la pesée à chaude par application d'une réfraction théorique de $2 \%$ pour les bovins, les ovins et les caprins. Le rendement brut a été évalué par la formule (poids carcasse/poids vif) $\times 100$. Le poids vif vide (PVV) a été calculé comme la différence entre le poids vif à jeun avant abattage et après éviscération (évacuation du contenu du tube digestif) ; le rendement de carcasse vrai a été calculé comme le rapport entre le poids carcasse froide (PCF) et le poids vif vide (PV).

Mangalmé et $34 \%$ à Mongo. La proportion des femelles abattues a été plus importante que les mâles (Figure 1).

Figure 1: Proportion des ovins abattus suivant le sexe dans les abattoirs municipaux de Mangalmé et Mongo dans la Province de Guera 
Dans la Province de Guera, les brebis ont été les plus abattues contrairement aux béliers sans la différence significative $(p>0,05)$. Parmi les femelles abattues 46 brebis ont été gravides dont 6 brebis issues de l'élevage sédentaire et 40 brebis de l'élevage transhumant. La proportion des brebis gravides a été non négligeable (Figure 2).

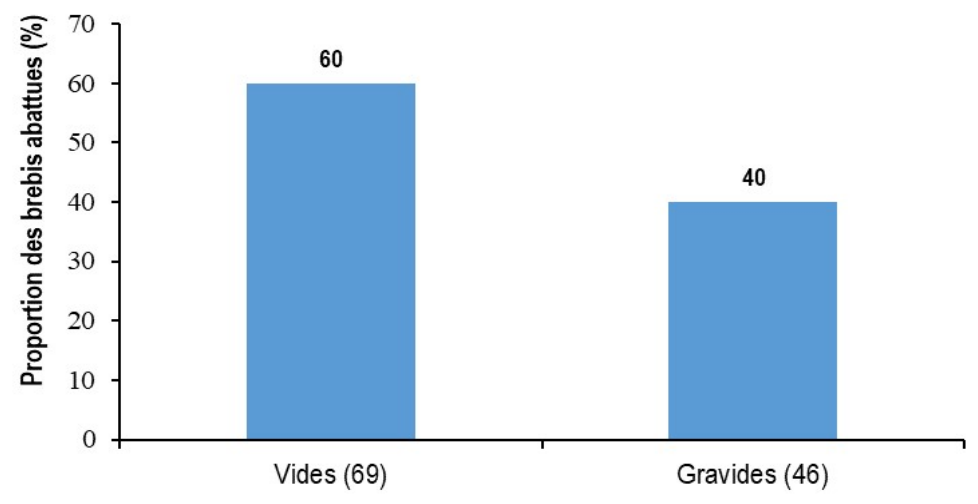

Catégories des brebis abattues

Figure 2: Proportion des femelles gravides abattues dans les abattoirs de Mangalmé et Mongo dans le Guera au Tchad

La proportion des brebis gravides abattues a été plus faible que celle des brebis vides sans différence significative $(p>0,05)$. Les ovins abattus dans le Guera proviennent de deux systèmes d'élevage (Figure 3).

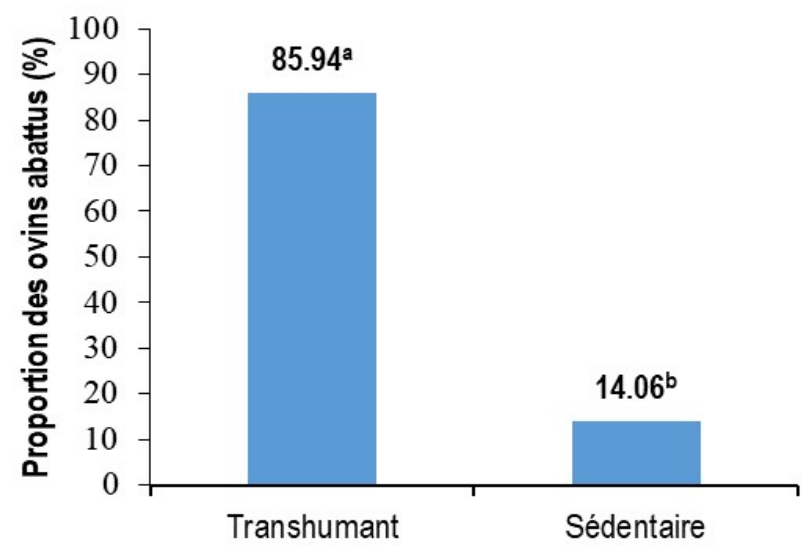

Système d'élevage des ovins abattus

Figure 3: Origine des ovins abattus dans les abattoirs municipaux de Mangalmé et Mongo dans la Province de Guera

L'élevage transhumant constitue la principale source d'approvisionnement en viande ovine des centres urbains de la Province de Guera $(p<0,05)$.
Poids vif à l'abattage : Le poids vif moyen des ovins à l'abattage de 29,13 $\pm 8,44 \mathrm{~kg}$ pour un âge moyen de $36,99 \pm 27,99$ mois. Ces paramètres ont varié suivant le sexe des ovins abattus (Tableau 2).

Tableau 2 : Age et poids moyens des ovins abattus dans la province du Guera, au centre du Tchad

\begin{tabular}{llll}
\hline Sexe & Age (mois) & Poids vif $(\mathbf{k g})$ & Effectif $(\mathbf{n})$ \\
\hline Mâle & $28,9 \pm 22,6^{\mathrm{a}}$ & $24,1 \pm 8,2^{\mathrm{a}}$ & 77 \\
Femelle & $42,4 \pm 31,6^{\mathrm{b}}$ & $32,5 \pm 8,6^{\mathrm{b}}$ & 115 \\
\hline Moyenne & $36,99 \pm 27,99$ & $29,13 \pm 8,44$ & 192 \\
\hline
\end{tabular}

Les colonnes affectées des lettres différentes sont significativement différentes $(p<0,05)$ 

du Tchad.

Les béliers abattus ont été plus jeunes avec un poids plus faible contrairement aux brebis qui ont été plus lourdes avec un âge plus avancé $(p<0,05)$. Le poids vif et l'âge à l'abattage ont également varié suivant l'origine des ovins (Tableau 3).

Tableau 3. Poids vif $(\mathrm{kg})$ et âges (mois) moyens des ovins abattus à Mangalmé et à Mongo au Centre du Tchad selon le sexe et le type d'élevage

\begin{tabular}{lcccc}
\hline \multirow{2}{*}{ Origine } & \multicolumn{2}{c}{ Femelles } & \multicolumn{2}{c}{ Mâles } \\
\cline { 2 - 5 } & Poids vif (kg) & Age (mois) & Poids vif (kg) & Age (mois) \\
\hline Sédentaire & $32,6 \pm 8,2^{\mathrm{a}}$ & $45,7 \pm 40,1^{\mathrm{a}}$ & $18,8 \pm 6,7^{\mathrm{a}}$ & $14,0 \pm 8,2^{\mathrm{a}}$ \\
Transhumant & $32,4 \pm 8,7^{\mathrm{a}}$ & $42,1 \pm 31,0^{\mathrm{a}}$ & $25,3 \pm 8,1^{\mathrm{b}}$ & $32,6 \pm 23,5^{\mathrm{b}}$ \\
\hline Moyenne & $32,26 \pm 8,63$ & $42,61 \pm 32,28$ & $24,39 \pm 7,90$ & $29,98 \pm 21,35$ \\
\hline
\end{tabular}

Les colonnes affectées des lettres différentes sont significativement différentes $(p<0,05)$

Les béliers issus des élevages transhumants ont été significativement plus lourds et âgés que ceux issus des élevages sédentaires $(p<0,05)$. Cette différence n'a pas été rapportée chez les brebis entre les deux systèmes d'élevage $(p>0,05)$.

Poids carcasse à chaud: Le poids carcasse moyen à chaud a été de $15,37 \pm 5,77 \mathrm{~kg}$, et a varié suivant le sexe des ovins abattus: les carcasses femelles ont été les plus lourdes $(17,8 \pm 6,3 \mathrm{~kg})$ que celles des mâles plus légères $(13,2 \pm 5,3 \mathrm{~kg})(p<0,05)$. Ce poids a également varié suivant le type d'élevage ou leur provenance (Tableau 4).

Tableau 4 : Poids carcasse moyen $(\mathrm{kg})$ des ovins abattus dans la Province du Guera au Centre du Tchad

\begin{tabular}{lcc}
\hline Origine & Femelle & Mâle \\
\hline Elevage sédentaire & $17,8 \pm 4,4^{\mathrm{a}}$ & $11,1 \pm 3,3^{\mathrm{a}}$ \\
Elevage transhumant & $17,8 \pm 6,5^{\mathrm{a}}$ & $13,7 \pm 5,5^{\mathrm{a}}$ \\
\hline Moyenne & $17,8 \pm 6,3$ & $13,2 \pm 5,3$ \\
\hline
\end{tabular}

Les colonnes affectées des lettres différentes sont significativement différentes $(p<0,05)$.

L'origine des ovins abattus n'a pas eu d'effet significatif sur le poids à l'abattage $(p>0,05)$. Le poids carcasse de brebis a été plus élevé que celui des béliers. Suivant l'origine des ovins, la différence de moyenne n'a pas significativement varié entre les ovins transhumants et sédentaires $(p>0,05)$.

Rendement carcasse à froid : Le rendement carcasse moyen a été de $54,42 \pm 12,10 \%$ et a varié suivant le sexe et l'origine des ovins abattus (Tableau 5).

Tableau 5: Rendement carcasse moyen (\%) à froid des ovins abattus dans la province du Guera, au Centre-Est du Tchad

\begin{tabular}{lcc}
\hline Origine & Femelles & Mâles \\
\hline Elevage sédentaire & $53,9 \pm 7,0^{\mathrm{a}}$ & $59,0 \pm 38,9^{\mathrm{a}}$ \\
Elevage transhumant & $53,7 \pm 11,0^{\mathrm{a}}$ & $54,6 \pm 15,2^{\mathrm{b}}$ \\
\hline Moyenne & $53,7 \pm 10,7$ & $55,5 \pm 14,2$ \\
\hline
\end{tabular}

Les colonnes affectées des lettres différentes sont significativement différentes $(p<0,05)$.

Les ovins sédentaires ont eu un rendement carcasse supérieur par rapport aux ovins transhumants avec une différence significative pour les mâles $(p<0,05)$ et non significative pour les femelles $(p>0,05)$. 


\section{DISCUSSION}

Effectifs, sexe et origine des ovins abattus: Les rendements carcasse des ovins abattus dans les villes de Mongo et Mangalmé ont été estimés. La contribution des ovins transhumants dans l'approvisionnement en viande de ces deux villes a été conséquente. En fonction de sexe des ovins abattus, les brebis ont représenté une part plus importante que les béliers. Les mêmes observations sur l'abattage élevé des brebis par rapport aux béliers ont été rapportés chez les caprins (Mopaté et al., 2019). Ce qui laisser présager d'une perte énorme en reproduction des brebis et des agneaux. II serait nécessaire de prendre de disposition pouvant limiter l'abattage des brebis à l'exception de celles de reformes. Selon l'origine des ovins abattus dans la province du Nord-Guéra, on est en droit d'affirmer que l'approvisionnement de cette zone en viande des petits ruminants est surtout assuré par les animaux provenant des élevages transhumants. Cela est en accord avec les observations de Mopaté et al. (2019) qui rapportent que les caprins abattus dans la même province proviennent à plus de $85 \%$ des élevages transhumants. En effet, cette province est un point de transit des éleveurs transhumants en provenance des autres provinces du Tchad telles que le Batha, Biltine ou Ouaddaï, en route pour celles de Salamat et de Moyen-Chari. Plusieurs de ces éleveurs y passent parfois la saison de pluies sur le chemin de retour. II n'est donc pas exclu, qu'ils puissent vendre des ovins pour leur subsistance.

Poids vif et l'âge à l'abattage: L'âge d'abattage des ovins dans la province du Guera a été de trois ans avec un poids vif d'environ $30 \mathrm{~kg}$. Le poids à l'abattage a été le même que celui rapporté par DSPA (2011), inférieur à $43,94 \mathrm{~kg}$ chez les ovins sahéliens mais supérieur à 23,43 $\mathrm{kg}$ chez les Djallonké rapportés au Benin par Djenontin et al. (2017).Considérant le sexe des ovins, les brebis ont été les plus âgés et plus lourdes à l'abattage par rapport aux béliers. La différence de poids et l'âge des brebis à l'abattage n'a pas été significative selon leur origine. Contrairement aux brebis, le poids moyen et l'âge des béliers transhumants à l'abattage ont été significativement plus élevés que ceux sédentaires. L'âge moyen élevé des mâles provenant des ovins transhumants laisse croire à une réforme de certains reproducteurs. Les mâles abattus ont plus de 24 mois. Le poids moyen supérieur des mâles transhumants s'expliquerait par l'âge moyen élevé de ces ovins. Chez les femelles vides, il est aussi probable qu'on ait à faire aux animaux de réforme car ce sont des femelles âgées de plus de 45 mois qui ont été abattues.
Poids carcasse à chaud : Le poids carcasse de brebis plus élevé que celui des béliers, s'explique par le fait que les béliers sont abattus à un âge plus faible que les brebis. Ce poids n'a pas été différent de 15,67 $\pm 3,16 \mathrm{~kg}$ rapporté chez le mouton sahélien mais supérieur à 12,87 $\pm 3,33 \mathrm{~kg}$ chez le Djallonké du Niger (Sangaré et al., 2005). Considérant l'origine des ovins abattus, les brebis des élevages transhumants ont eu de poids carcasse comparable à celle des élevages sédentaires. En revanche, pour les béliers, les ovins des élevages sédentaires ont été légèrement plus lourds que ceux transhumants sans différence significative. Toutefois, cette légère supériorité se traduit par le niveau d'engraissement suite à la valorisation des ressources pastorale diverse du parcours naturel lors de la mobilité du troupeau.

Rendement carcasse: Le rendement carcasse des ovins sahélien dans la zone d'étude a été appréciable. Les femelles ont eu un rendement carcasse faible comparé à celui des mâles. Ce résultat confirme les observations rapportées chez les caprins dans les mêmes villes. Ainsi, il est judicieux d'abattre les mâles pour leur meilleur rendement carcasse par rapport aux femelles. D'ailleurs l'abattage des femelles gravides est de nature à entrainer des pertes en reproduction (femelles et petits). Ces pertes sont souvent négligées ou ignorées alors qu'elles sont importantes. Elles peuvent avoir un impact négatif sur le renouvellement des ovins et affecter négativement la production durable de viande des petits ruminants à long termes (Mopaté et al., 2019; Nana et al., 2014 ; Alade et al., 2011; Garba et al., 1992). D'ailleurs, Nana et al. (2014) précisent que si les femelles gravides sont épargnées de l'abattage, l'effectif des petits ruminants pourrait augmenter de $3,2 \%$. Cet écart entre le sexe place le rendement carcasse des mâles au-dessus de celui de femelles alors que les femelles ont été les plus abattues avec un taux d'approvisionnement de $60 \%$. S'agissant de l'origine des ovins abattus dans la province du Guera, le rendement carcasse des femelles n'ont pas eu de différence significative entre élevages transhumant et sédentaire. En revanche, les béliers sédentaires ont eu un rendement carcasse meilleur $(59,0 \pm 38,9 \%)$ que ceux transhumants $(54,6 \pm 15,2 \%)$. Le rendement carcasse a été légèrement inférieur à $55,04 \pm 0,87 \%$ que celui des ovins sahéliens du Benin (Alkoiret et al., 2007), supérieur à $39-40 \%$ chez les ovins Arabe et Peuls du Tchad (Dumas, 1980) et 44,9\% (valeur maximale) rapporté au Burkina suivant différents régiments alimentaires des ovins (Zoundi et al., 2002). 


\section{CONCLUSION ET APPLICATION DES RESULTATS}

L'étude a permis de déterminer les poids vif moyen (avant abattage), les poids carcasse et le rendement carcasse moyens dans la Province du Guera. II ressort que plus de $85 \%$ des ovins abattus dans ces deux villes proviennent des élevages transhumants et concerne surtout les femelles. Le rendement a été meilleur pour les béliers que les brebis et plus particulièrement pour les béliers sédentaires que les transhumants. Ainsi, l'abattage des béliers est à encourager pour limiter des pertes en reproduction des femelles et améliorer l'approvisionnement en viande de la population. Ce résultat suggère d'orienter et d'encourager les éleveurs dans la production des agneaux de boucherie. Pour cela une transition du système extensif transhumant à un mode d'élevage intensif et spécialisé est conseillée. Les brebis épargnées de l'abattage induiront une amélioration de la productivité des troupeaux. Ce mode d'élevage permettra également de mieux valoriser les ressources alimentaires locales, d'améliorer la qualité de la viande produite et de maîtriser la reproduction ovine en zone sahélienne du Tchad.

\section{REMERCIEMENTS}

Les auteurs remercient les autorités administratives en charge des abattoirs et les bouchers qui ont permis la réalisation de ce travail.

\section{REFERENCES BIBLIOGRAPHIQUES}

Alade NK, Sadisu MA, Gambo M. 2011. Incidence of slaughtering pregnant cows, sheep, goats and camels in a Sahel Region of Nigeria. Research Opinions in Animal \& Veterinary Sciences (ROAVS), 1(8), 516-520.

Alary V, Aboul-Naga A, El Shafie M, Abdelkrim N, Hamdon H, Metawi H, 2015. Roles of small ruminants in rural livelihood improvement Comparative analysis in Egypt. Rev. Elev. Med. Vet. Pays Trop., 68 (2-3): 79-85.

Alkoiret TI, Soule Manne AA, Gbangboche AB, Attakpa $E Y$, 2007. Performances d'embouche des ovins Djallonké complémentés avec les coques de graine de coton au Bénin. Livestock Research for Rural Development. Volume 19, Article \#141. Retrieved September 24, 2019, from http://www.Irrd.org//lrd19/10/alko19141.htm.

DESPA (Direction des Études, des Statistiques, de la Programmation et des Archives), 2011. Rapport annuel des statistiques. Ministère du Développement Pastoral et des Productions Animales, $52 \mathrm{p}$.

Djenontin J, Senou M, Salifou CFA, Ahounou GS, Issifou TM, Youssao AKI, 2017. Influence du type génétique sur la composition corporelle et la qualité de la viande des ovins élevés dans les systèmes d'élevage traditionnels. Arch. Zootec. 66 (256): 523-533.

Dumas R, 1980. Contribution à l'étude des petits ruminants du Tchad. Rev. Elev. Méd. Vét. Pays trop., 33 (2) : $215-233$.

Garba HS, Hasan WA, Akingbemi BT, 1992. Foetal wastage through slaughtering of pregnant cattle at the Sokoto abattoir. Tropical Veterinarian, 10:123- 126.

Idahor KO, 2013. Sheep and Goats Slaughtered At Keffi Abattoir: Health Status, Carcass Yield and Foetal Deaths. J. Anim. Sci. Adv. 3(6): 276-283. DOI:

http://dx.doi.org/10.5455/jasa.20130619102201.

Mopaté LY, Tellah M, Djalal AK, Souleyman MS, 2019. Carcass yield of Sahelian goats in Guera Province at central-eastern Chad. International Journal of Livestock Research, 9(9): 30-36. DOI: http://dx.doi.org/10.5455/ijlr.20190619074240.

Nana FCN, Tume C, Daouda, Djitie FK, Dandji MBS, Zoli AP, Beckers JF, 2014. Impact de l'abattage des chèvres gravides sur l'élevage des petits ruminants au Cameroun. Livestock Research for Rural Development. Volume 26, Article \#210. Retrieved September 27, 2019, from http://www.Irrd.org//rrd26/11/nana26210.html.

Sangaré M, Thys E, Gouro AS, 2005. L'alimentation des ovins de race locale: Techniques d'embouche ovine, choix de l'animal et durée. Fiche technique Production animale en Afrique de I'Ouest, CIRDES, N13.

Zoundi JS, Nianogo AJ, Sawadogo L, 2002. Utilisation optimale de ressources alimentaires localement disponibles pour l'engraissement des ovins au sein des exploitations mixtes agriculture-élevage du plateau central du Burkina Faso. Revue Élev. Méd. vét. Pays trop., 55 (1) : 53-62. 